\title{
Basic Amino Acids as Green Catalysts for Isomerization of Glucose to Fructose in Water
}

\author{
Qiang Yang, Matthew Sherbahn, and Troy Runge* \\ Department of Biological Systems Engineering, University of Wisconsin-Madison, 460 Henry \\ Mall, Madison, WI 53706
}

SUPPLEMENTARY INFORMATION (PAGES S1-19)

CONTENTS (contains 3 schemes, 5 tables and 10 figures)

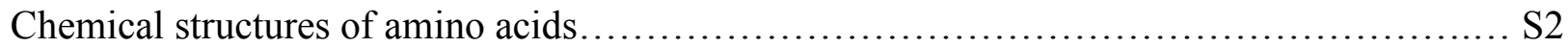

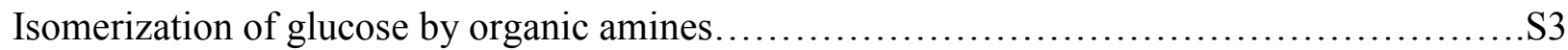

Effects of dosage and reaction temperature on rate constant........................... 4

Effect of reaction temperature on rate constant for the isomerization of glucose by lysine......S5

Estimated equilibrium conversion and constant for glucose isomerization...................S6

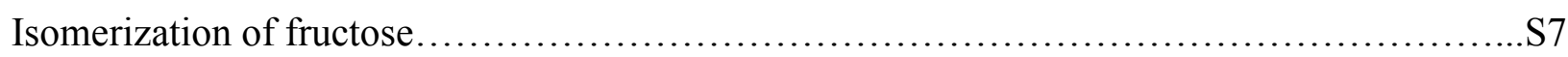

Effect of reaction temperature on rate constant and calculated apparent activation energy for

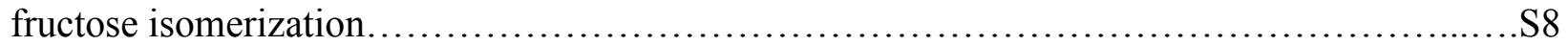

Estimated equilibrium conversion and constant for fructose isomerization...................S9

Plot of $\ln \kappa$ as a function of $1 / \mathrm{T}$ for fructose isomerization................................... 10

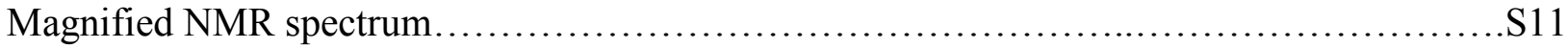

Illustration of reaction between amine and water...................................... 12

Effect of DL-glyceraldehyde on the isomerization of glucose .......................... 13

Effects of glyceraldehyde on kinetics and catalytic performance for glucose isomerization.....S14

${ }^{1} \mathrm{H}-{ }^{13} \mathrm{C}$ HSQC NMR spectra of glucose-2-D, glucose, mannose, fructose and arginine......S15-17 Isomerization of glucose or glucose-2-D in $\mathrm{D}_{2} \mathrm{O}$ and in

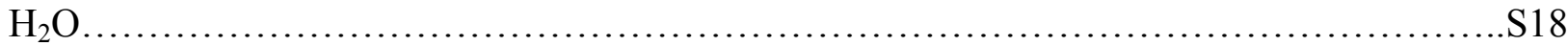
Illustration of possible pathways for isomerization, decomposition and condensation reactions.

*To whom correspondence should be addressed. Tel: 608-890-3143. E-mail: trunge@wisc.edu 

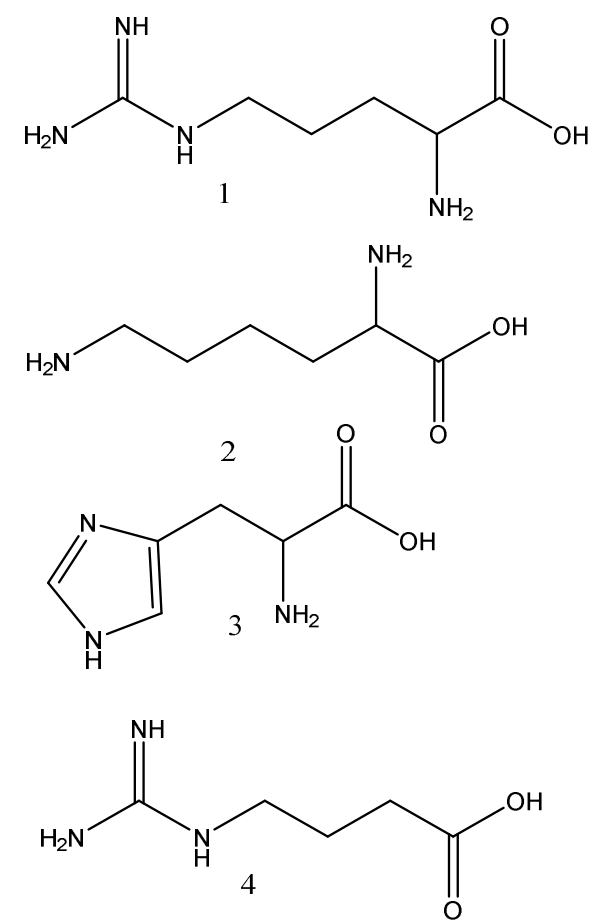<smiles>N=C(N)NCCC(=O)O</smiles><smiles>N=C(N)NCC(=O)O</smiles>

Scheme S1. Chemical structures of arginine (1), lysine (2), histidine (3), 4-guanidinobutyric acid (4), 3-guanidinopropionic acid (5) and guanidineacetic acid (6). 


\section{Table S1. Isomerization of Glucose by Organic Amines}

\begin{tabular}{|c|c|c|c|c|c|c|}
\hline organic amine & $\mathrm{pKa}$ & $\begin{array}{l}\text { dosage } \\
(\mathrm{mol} \%)\end{array}$ & $\begin{array}{l}\text { temp } \\
\left({ }^{\circ} \mathrm{C}\right)\end{array}$ & $\begin{array}{l}Y_{\text {Fru }} \\
(\%)\end{array}$ & $\begin{array}{l}\mathrm{S}_{\text {Fru }} \\
(\%)\end{array}$ & Ref. \\
\hline triethylamine & 10.8 & $\begin{array}{l}10 \\
5 \\
2\end{array}$ & $\begin{array}{c}120 \\
110 \\
80 \\
60 \\
120 \\
100 \\
80 \\
100 \\
100\end{array}$ & $\begin{array}{l}19 \\
28 \\
24 \\
14 \\
28 \\
32 \\
28 \\
27 \\
23\end{array}$ & $\begin{array}{l}35 \\
56 \\
45 \\
52 \\
49 \\
63 \\
63 \\
61 \\
74\end{array}$ & 1 \\
\hline $\begin{array}{l}\text { morpholine } \\
\text { piperazine } \\
\text { ethylenediamine } \\
\text { piperidine } \\
\text { pyrrolidine }\end{array}$ & $\begin{array}{l}8.4 \\
9.8 \\
10.8 \\
11.2 \\
11.3\end{array}$ & $\begin{array}{l}10 \\
10 \\
10 \\
10 \\
10\end{array}$ & $\begin{array}{l}100 \\
100 \\
100 \\
100 \\
100\end{array}$ & $\begin{array}{l}17 \\
28 \\
25 \\
29 \\
29\end{array}$ & $\begin{array}{l}43 \\
62 \\
60 \\
51 \\
59\end{array}$ & 2 \\
\hline imidazole & 7.05 & 160 & $\begin{array}{c}80 \\
100 \\
120\end{array}$ & $\begin{array}{l}10 \\
29 \\
33\end{array}$ & $\begin{array}{l}40 \\
54 \\
55\end{array}$ & \\
\hline 1-(3-aminopropyl) imidazole & $9.63,6.5$ & 7.5 & $\begin{array}{c}80 \\
100 \\
120\end{array}$ & $\begin{array}{l}12 \\
18 \\
20\end{array}$ & $\begin{array}{l}41 \\
54 \\
43\end{array}$ & \\
\hline tetramethylguanidine & 13.6 & 3 & $\begin{array}{c}80 \\
100 \\
120\end{array}$ & $\begin{array}{l}33 \\
36 \\
30\end{array}$ & $\begin{array}{l}73 \\
71 \\
51\end{array}$ & 3 \\
\hline 1,5,7-triazabicyclo[4.4.0]dec-5-ene & 21 & 3 & $\begin{array}{c}80 \\
100 \\
120\end{array}$ & $\begin{array}{l}31 \\
32 \\
32\end{array}$ & $\begin{array}{l}65 \\
67 \\
50\end{array}$ & \\
\hline $\operatorname{tris}(2$-aminoethyl)amine & $\begin{array}{r}10.24,9.43 \\
8.45,2.60\end{array}$ & 3 & 100 & 24 & 56 & \\
\hline
\end{tabular}

\section{References}

1. Carraher, J.M.; Fleitman, C.N.; Tessonnier, J.P. ACS Catal. 2015, 5, 3162-3173.

2. Liu, C.; Carraher, J.M.; Swedberg, J.L.; Herndon, C.R.; Fleitman, C.N; Tessonnier, J.P. ACS Catal. 2014, 4, 4295-4298.

3. Yang, Q.; Zhou, S.F.; Trunge, T. J. Catal. 2015, 330, 474-484. 


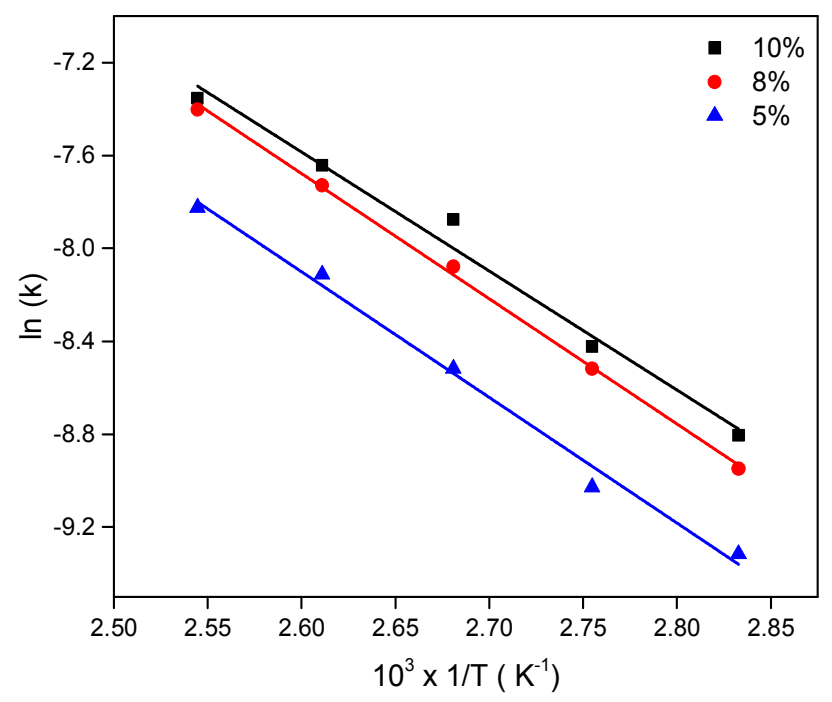

Figure S1. Plots of $\ln \kappa$ as a function of $1 / \mathrm{T}$ under different concentrations for the isomerization of glucose by arginine. Reaction conditions: $10 \mathrm{wt} \%$ glucose, $5 \mathrm{~mol} \%$ or $8 \mathrm{~mol} \%$ or $10 \mathrm{~mol} \%$ arginine relative to glucose, $2-20 \mathrm{~min}, 1 \mathrm{~mL} \mathrm{H}_{2} \mathrm{O}, 80-120^{\circ} \mathrm{C}$. 


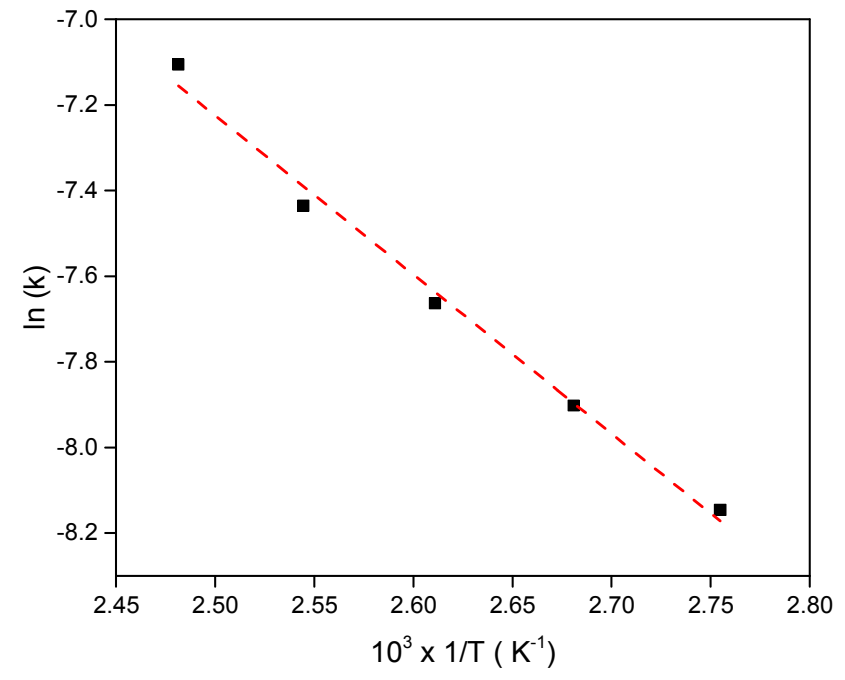

Figure S2. Plot of $\ln \kappa$ as a function of $1 / \mathrm{T}$ for the isomerization of glucose by lysine. Reaction conditions: $10 \mathrm{wt} \%$ glucose, $20 \mathrm{~mol} \%$ lysine relative to glucose, $2-20 \mathrm{~min}, 1 \mathrm{~mL} \mathrm{H}_{2} \mathrm{O}, 90-130{ }^{\circ} \mathrm{C}$. 
Table S2. Estimated Equilibrium Conversion and Constant for Glucose Isomerization by Arginine

\begin{tabular}{|c|c|c|c|}
\hline $\begin{array}{l}\text { dosage } \\
\%\end{array}$ & $\begin{array}{l}\text { temp } \\
\left(^{\circ}\right)\end{array}$ & $\begin{array}{l}\text { equilibrium constant } \\
\qquad K_{e q}\end{array}$ & $\begin{array}{c}\text { equilibrium conversion } \\
\qquad \%\end{array}$ \\
\hline \multirow{5}{*}{10} & 120 & 0.54 & 35.1 \\
\hline & 110 & 0.42 & 29.6 \\
\hline & 100 & 0.28 & 21.9 \\
\hline & 90 & 0.21 & 17.3 \\
\hline & 80 & 0.17 & 14.5 \\
\hline \multirow{5}{*}{8} & 120 & 0.49 & 32.9 \\
\hline & 110 & 0.35 & 25.9 \\
\hline & 100 & 0.27 & 21.2 \\
\hline & 90 & 0.15 & 13.0 \\
\hline & 80 & 0.07 & 6.5 \\
\hline \multirow{5}{*}{5} & 120 & 0.38 & 27.5 \\
\hline & 110 & 0.26 & 20.6 \\
\hline & 100 & 0.21 & 17.3 \\
\hline & 90 & 0.12 & 10.7 \\
\hline & 80 & 0.05 & 4.8 \\
\hline
\end{tabular}

Equilibrium constant $\left(K_{\text {eq }}\right)$ was calculated from the ratio of [fructose concentration at 15 $\mathrm{min}] /[$ glucose concentration at $15 \mathrm{~min}]$. Equilibrium conversion (\%) was calculated by the equation: $K_{e q} /\left(1+K_{e q}\right) \times 100 \%$. 

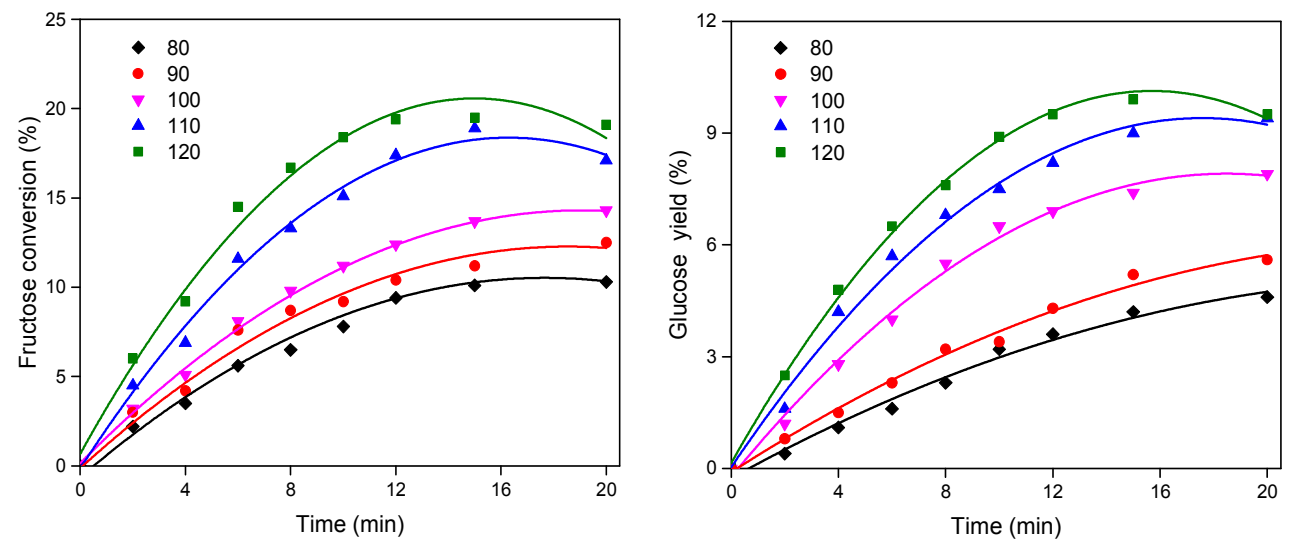

Figure S3. Isomerization of fructose by arginine. Reaction conditions: $10 \mathrm{wt} \%$ fructose, $8 \mathrm{~mol} \%$ arginine relative to fructose, $2-20 \mathrm{~min}, 1 \mathrm{~mL} \mathrm{H}_{2} \mathrm{O}, 80-120{ }^{\circ} \mathrm{C}$. 
Table S3. Effect of Reaction Temperature on Rate Constant and Calculated Apparent Activation Energy for Fructose Isomerization by Arginine

$\begin{array}{cccccc}\begin{array}{c}\mathrm{E}_{\mathrm{a}} \\ \left(\mathrm{kJ} \cdot \mathrm{mol}^{-1}\right)\end{array} & \begin{array}{c}\text { temp } \\ \left({ }^{\circ} \mathrm{C}\right)\end{array} & \begin{array}{c}10^{-4} \times \kappa \\ \left(\mathrm{s}^{-1}\right)\end{array} & \begin{array}{c}\mathrm{Y}_{\mathrm{Glu}} \\ (\%)\end{array} & \begin{array}{c}\mathrm{S}_{\mathrm{Glu}} \\ (\%)\end{array} & \begin{array}{c}10^{-3} \times \mathrm{TOF} \\ \left(\mathrm{mol}_{\mathrm{Glu}} \cdot \mathrm{mol}^{-1} \mathrm{Arg} \cdot \mathrm{s}^{-1}\right)\end{array} \\ & 120 & 2.7 & 10 & 50 & 1.38 \\ 28.7 & 110 & 2.3 & 9 & 47 & 1.25 \\ & 100 & 1.7 & 7 & 54 & 0.97 \\ & 90 & 1.4 & 5 & 46 & 0.69 \\ & 80 & 1.0 & 4 & 41 & 0.56\end{array}$

Reaction conditions: $10 \mathrm{wt} \%$ fructose, $8 \mathrm{~mol} \%$ arginine relative to fructose, $2-20 \mathrm{~min}, 1 \mathrm{~mL}$ $\mathrm{H}_{2} \mathrm{O}, 80-120^{\circ} \mathrm{C}$. 
Table S4. Estimated Equilibrium Conversion and Constant for Fructose Isomerization by Arginine

$\begin{array}{ccc}\text { temp } & \text { equilibrium constant } & \text { equilibrium conversion } \\ \left(^{\circ}\right) & K_{e q} & \% \\ 120 & 0.11 & 9.9 \\ 110 & 0.11 & 9.9 \\ 100 & 0.09 & 8.3 \\ 90 & 0.06 & 5.7 \\ 80 & 0.06 & 5.7\end{array}$

Equilibrium constant $\left(K_{e q}\right)$ was calculated from the ratio of [glucose concentration at 15 $\mathrm{min}] /[$ fructose concentration at $15 \mathrm{~min}$ ]. Equilibrium conversion (\%) was calculated by the equation: $K_{e q} /\left(1+K_{e q}\right) \times 100 \%$. 


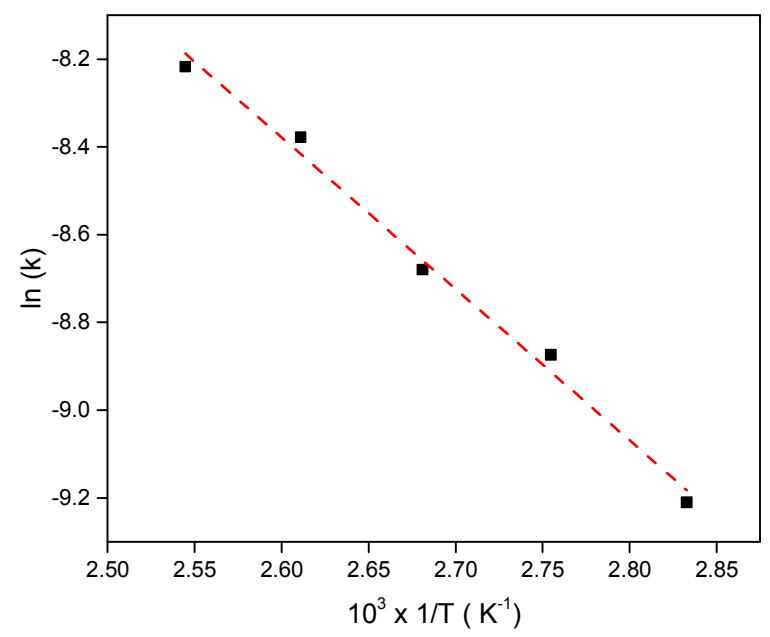

Figure S4. Plot of $\ln \kappa$ as a function of $1 / T$ for the isomerization of fructose by arginine. Reaction conditions: $10 \mathrm{wt} \%$ fructose, $8 \mathrm{~mol} \%$ arginine relative to fructose, $2-20 \mathrm{~min}, 1 \mathrm{~mL} \mathrm{H}_{2} \mathrm{O}$, $80-120^{\circ} \mathrm{C}$. 


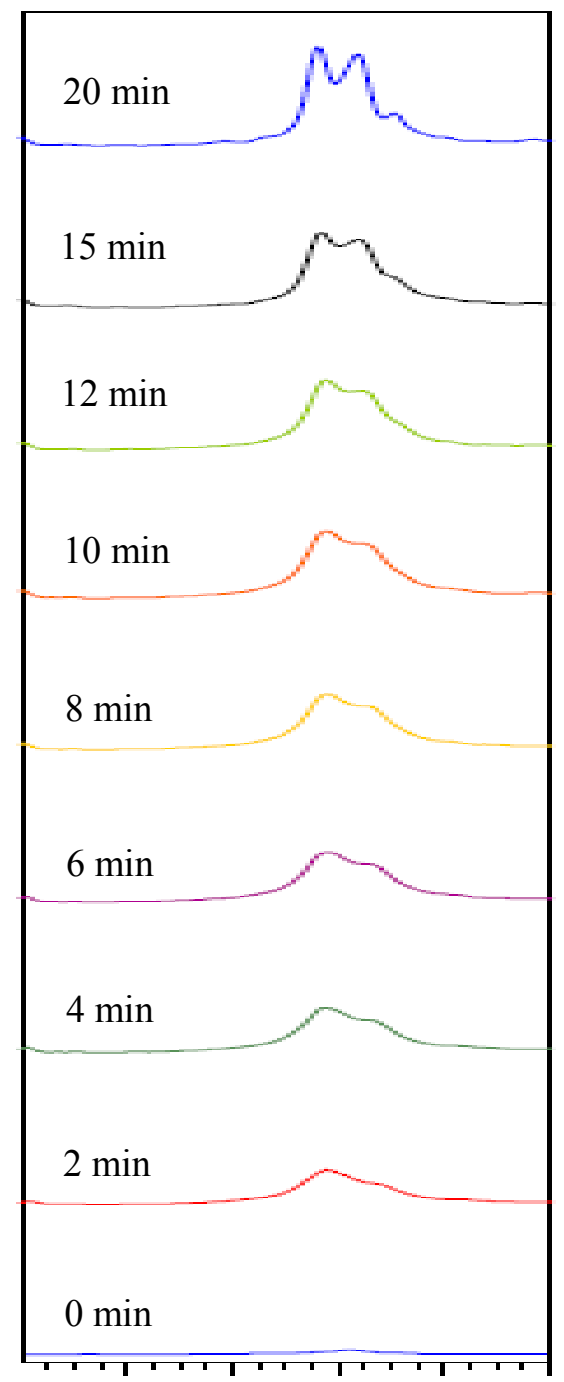

Figure S5. Change in intensity of characteristic peak around $\delta=3.92 \mathrm{ppm}$ attributed to formed fructose during glucose isomerization by arginine. 


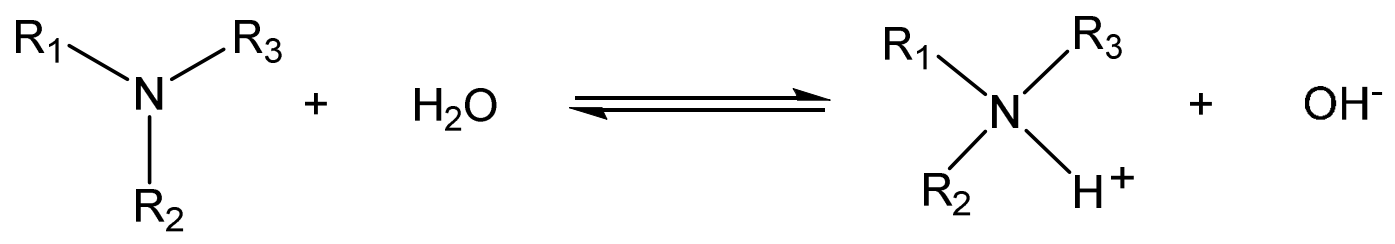

Scheme S2. Illustration of reaction between amine and water. 

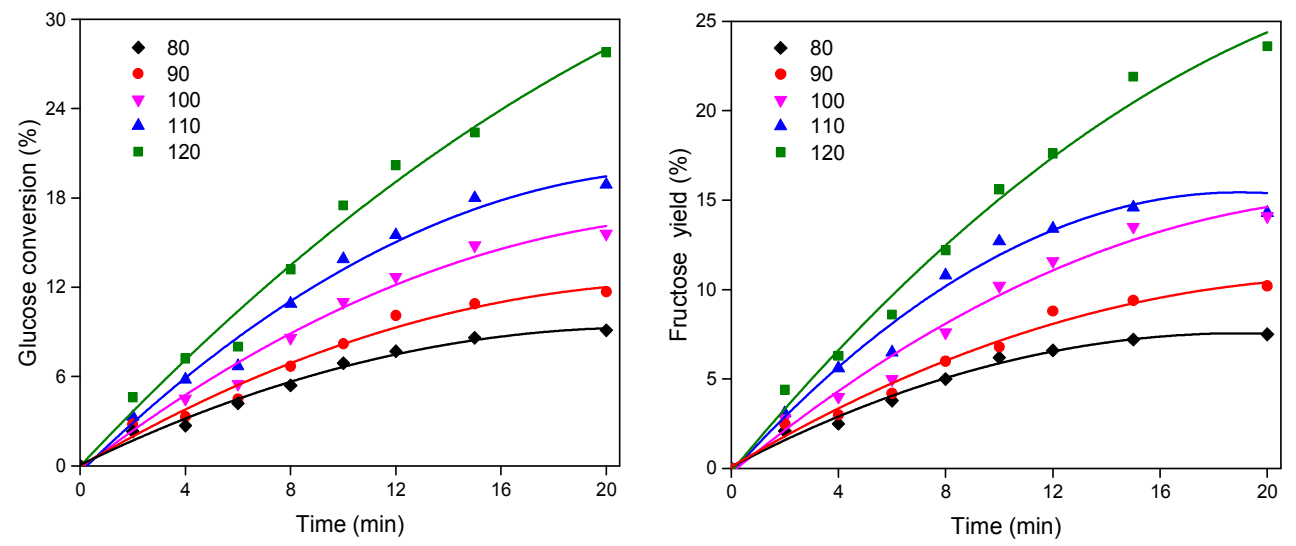

Figure S6. Effect of DL-glyceraldehyde on the isomerization of glucose by arginine. Reaction conditions: $10 \mathrm{wt} \%$ glucose, $8 \mathrm{~mol} \%$ arginine relative to glucose, $8 \mathrm{~mol} \%$ DL-glyceraldehyde relative to glucose, $2-20 \mathrm{~min}, 1 \mathrm{~mL} \mathrm{H}_{2} \mathrm{O}, 80-120{ }^{\circ} \mathrm{C}$. 
Table S5. Effects of Addition of Glyceraldehyde on Kinetics and Catalytic Performance for Glucose Isomerization by Arginine

$\begin{array}{cccc}\text { temp } & 10^{-4} \times \kappa & Y_{\text {Fru }} & S_{\text {Fru }} \\ \left({ }^{\circ} \mathrm{C}\right) & \left(\mathrm{s}^{-1}\right) & (\%) & (\%) \\ 120 & 3.1 & 23 & 85 \\ 110 & 2.4 & 14 & 75 \\ 100 & 1.8 & 14 & 91 \\ 90 & 1.4 & 10 & 87 \\ 80 & 1.0 & 7 & 82\end{array}$

Reaction conditions: $10 \mathrm{wt} \%$ glucose, $8 \mathrm{~mol} \%$ arginine relative to glucose, $8 \% \mathrm{~mol}$ DLglyceraldehyde relative to glucose, 2-20 min, $1 \mathrm{~mL} \mathrm{H}_{2} \mathrm{O}, 80-120{ }^{\circ} \mathrm{C}$. 

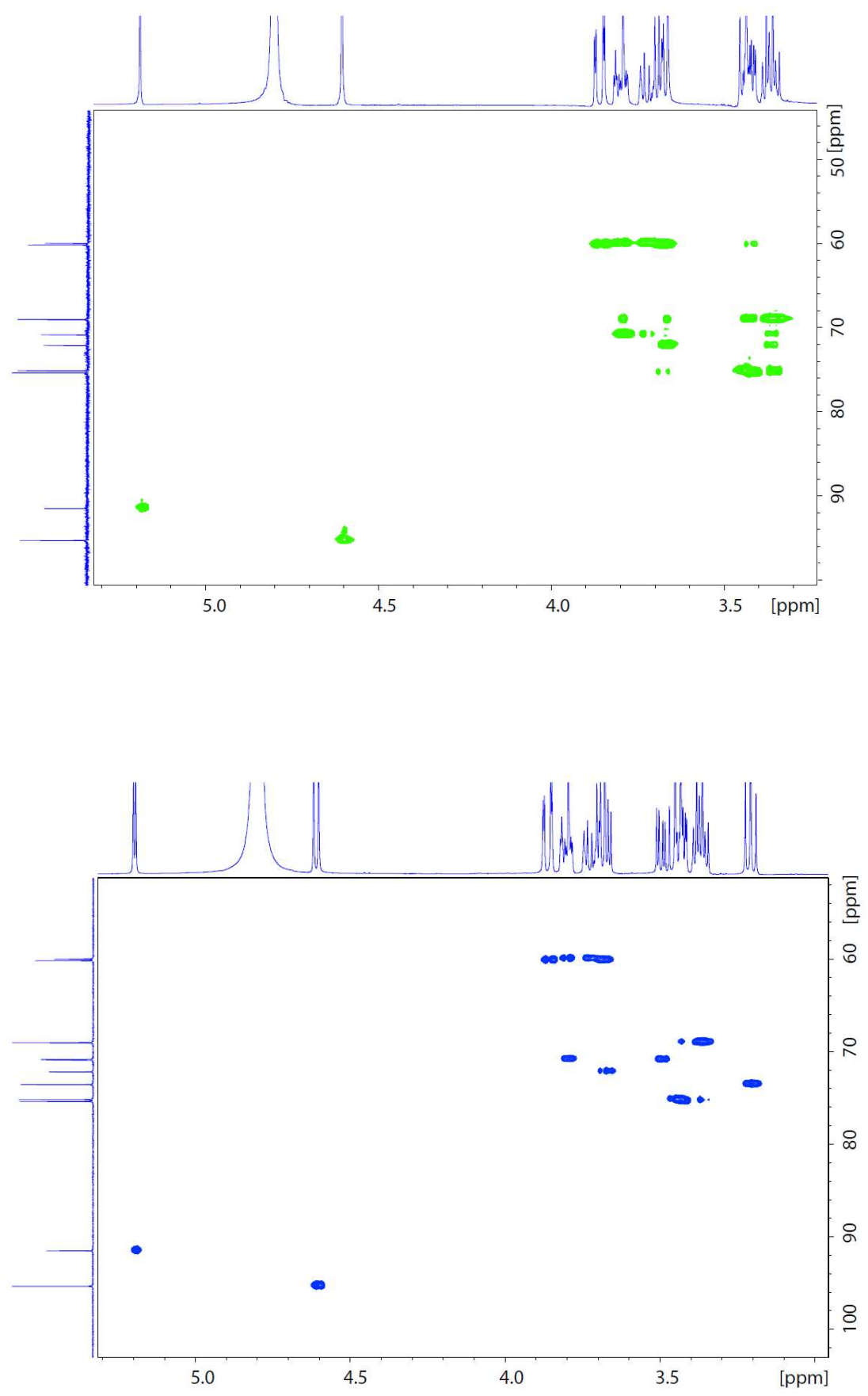

Figure S7. ${ }^{1} \mathrm{H}-{ }^{13} \mathrm{C}$ HSQC NMR spectra of glucose-2-D (top) and glucose (bottom). 

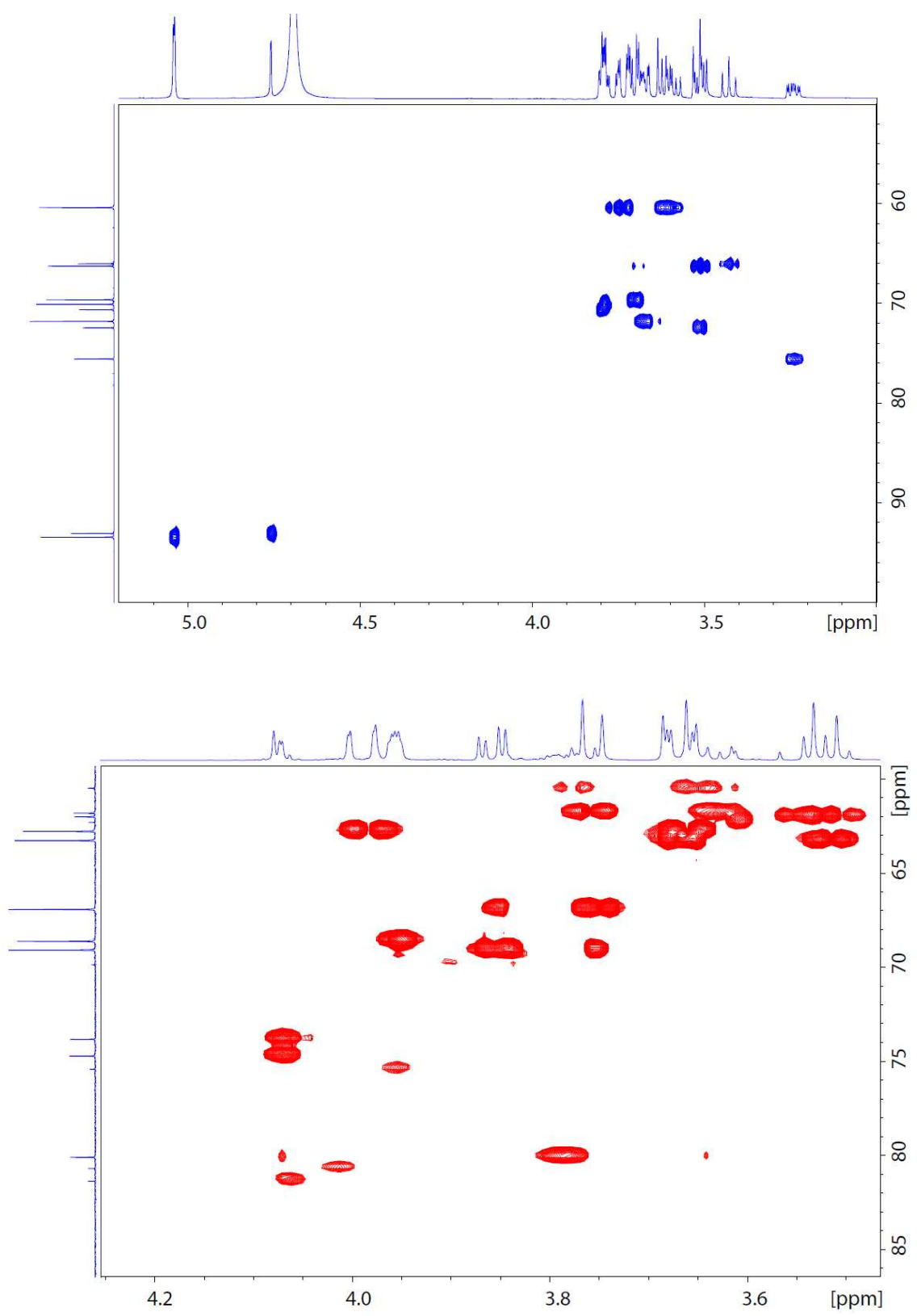

Figure S8. ${ }^{1} \mathrm{H}^{-13} \mathrm{C}$ HSQC NMR spectra of mannose (top) and fructose (bottom). 


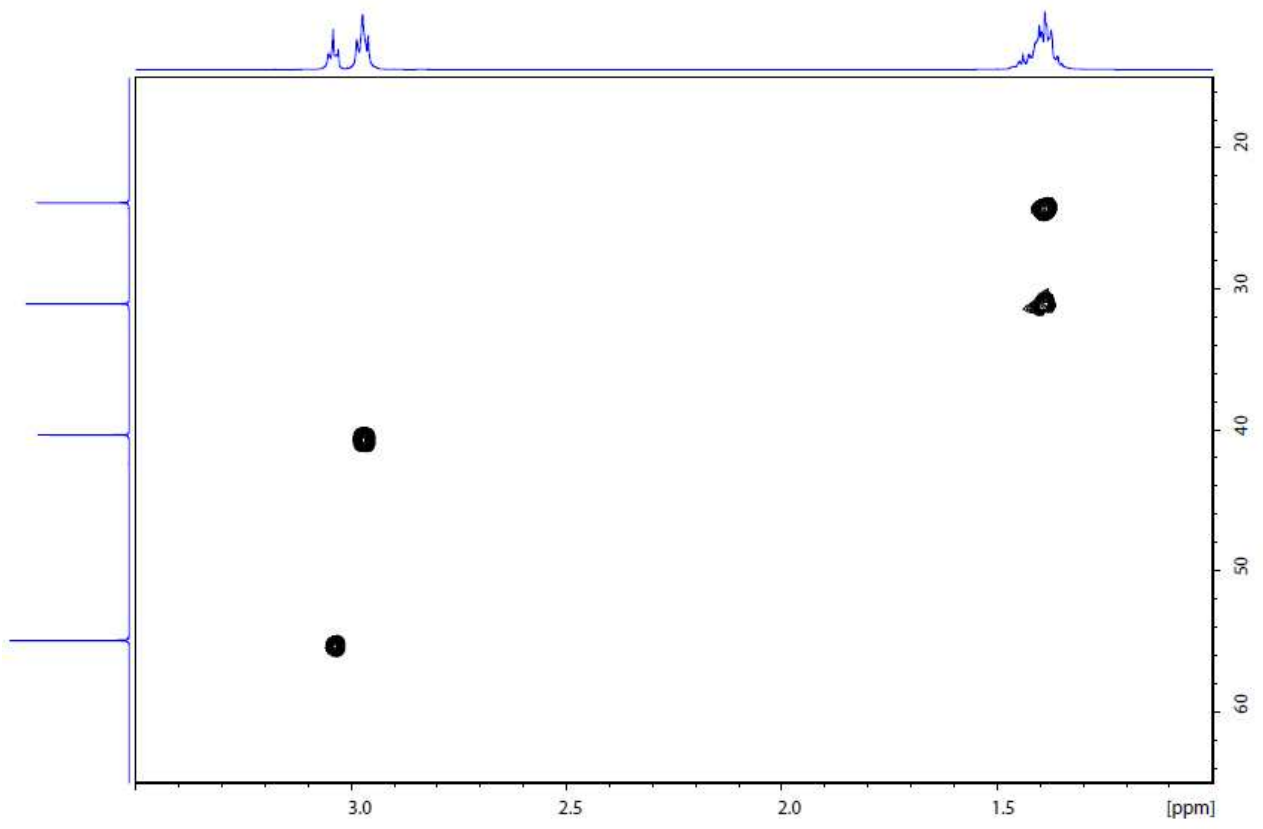

Figure S9. ${ }^{1} \mathrm{H}-{ }^{13} \mathrm{C}$ HSQC NMR spectrum of arginine. 


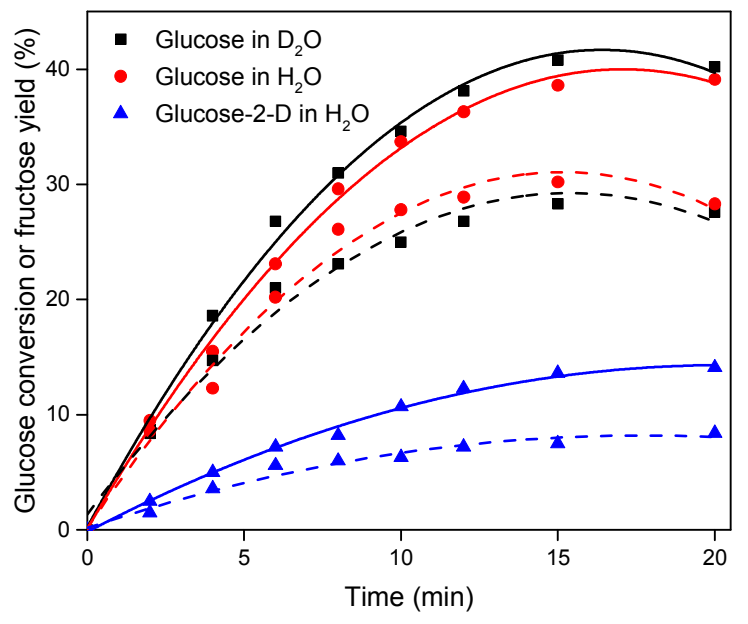

Figure S10. Isomerization of glucose or glucose-2-D by arginine in $\mathrm{D}_{2} \mathrm{O}$ and in $\mathrm{H}_{2} \mathrm{O}$. Solid line: glucose conversion. Dash line: fructose yield. Reaction conditions: $10 \mathrm{wt} \%$ glucose or glucose-2$\mathrm{D}, 8 \mathrm{~mol} \%$ arginine relative to glucose or glucose-2-D, 2-20 min, $1 \mathrm{~mL} \mathrm{H}_{2} \mathrm{O}$ or $\mathrm{D}_{2} \mathrm{O}, 120{ }^{\circ} \mathrm{C}$. 


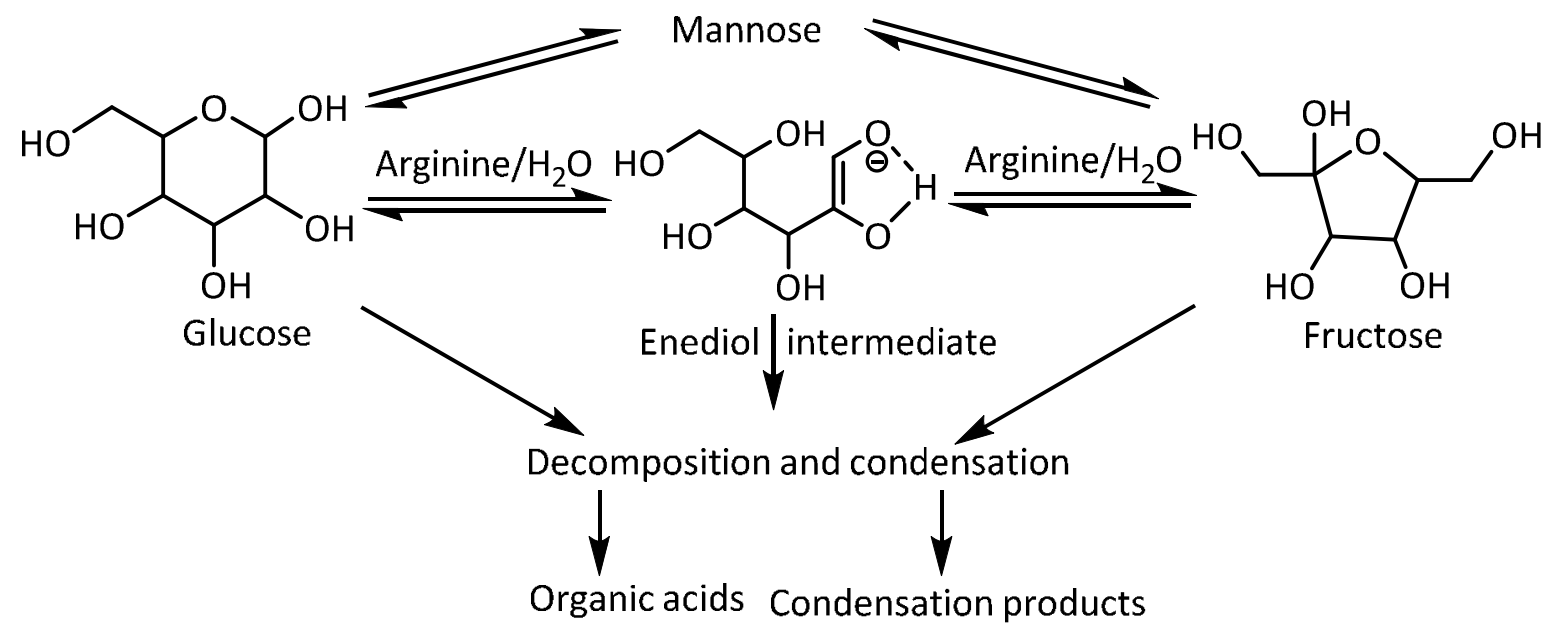

Scheme S3. Illustration of possible pathways for isomerization, decomposition and condensation reactions. 